\title{
Design And Analysis of New Compound Journal Bearing
}

\author{
Nadhim. $\mathrm{N}^{1}$, Mahmood. $\mathrm{R}^{2}$ \\ \{Nadhim.naseer@mtu.edu.iq ${ }^{1}$, Mahmech2001@yahoo.com² $\}$ \\ Al-Nahrain University, College of Engineering, Mechanical Engineering Department, Iraq ${ }^{1}$ \\ Al-Nahrain University, College of Engineering, Prosthetics ${ }^{2}$
}

\begin{abstract}
One of the main challenges that are widely faced is the maintenance of the journal bearing requires extra time and effort. Therefore, in this work a new design of journal bearings system was developed with low friction which would help with: A) relatively higher load as it would create duplicated pressure and B) Due to the use of the Nano Lubrication as one of the new system's components, it will support an enhanced and persistent oil viscosity of the mechanical system which would support a mechanical system of a consistent temperature. To accomplish all the above, a mathematical model for the new journal bearing design was developed, the mathematical model was additionally resolved using Reynold's equation and using the finite difference method. A MATLAB code was also developed to solve the governing partial differential equation to find journal-bearing parameters. The preliminary results of this work indicated an increase of pressure value of $50 \%$ as compared with the traditional bearing. Furthermore, the new design indicated a consistent oil viscosity/temperature when adding the Nanoparticles to the system and improve all bearing parameters.
\end{abstract}

Keywords: Hydrodynamic journal bearing, Nano lubricants, Viscosity, Pressure distribution, Load carrying capacity.

\section{Introduction}

Journal bearings are a common engineering component that can be found in practically any machine. To achieve great efficiency and dependability, combustion engines and turbines rely almost entirely on journal bearings [1]. A journal bearing is made up of a revolving shaft (journal) and a stationary bush. The hydrodynamic film that supports the load is created between the shaft's moving surfaces and the bush's moving surfaces. Because the operating surfaces of such bearings are completely separated by a lubricating film, the friction coefficient is kept low, around 0.005 , and bearing failure is rare, occurring only after incorrect operation. The mechanism of lubrication is explained by Reynolds' hypothesis, which involves the formation of a viscous liquid film between the moving surfaces. The prerequisite is that the surfaces must move with enough velocity relative to each other to create such a film [1]. All hydrodynamic lubrication can be mathematically represented using an equation devised by Reynolds. Normal journal bearings have some drawbacks in many applications, such as cavity pressure, power loss, instability, and so on. To boost capacity and efficiency, a variety of designs have been proposed, including offset and preload bearings, multi-lobe bearings, and step bearings [2]. The effect of lubricant and improving its performance is studied by many researchers. In this regard is K. G. Binu et al. [3] employed $\mathrm{TiO} 2$ nanoparticles to manufacture nano lubrication as a lubricant additive with a particle concentration of 0.02 to investigate the static performance 
properties of Journal bearings in low shear states. For TiO2 nanolubricants, static characteristics such as pressure, load capacity, and friction force were simulated in this study. When comparing mild shear to high shear, the results show an increase in pressure and load-carrying capacity. Han et al. [4] produced a CFD result. For the parallel sliding surface with spherical dimples, it was demonstrated that the lubricant's load-carrying capacity improves while its friction coefficient drops.

\section{Theory}

In the present design, the traditional journal bearing is modified as a compound bearing with is consisted of; inner and outer journal bearing and as follows; the shaft is bored, and an inner bush is pressed to perform the inner journal while the shaft outer surface makes the outer journal with both housings as shown in Fig 1. To allow for oiling of the inner bush the housing support is supplied with oil entry consisted of two perpendicular holes. The journal geometry is a hollow cylinder in which the two-journal bearing is introduced [3]. The first is the inner between the inner bush and the central support study. The second is the outer between the outer surface and the bush at the support. In other words, two parallel journals bearing are introduced. It is to be noted that the inner. In this case, the inner journal operates in a reverse manner as compared with the outer where the bush is rotated and the journal is fixed, the outer journal is working as usual. However, since there exists a relative motion, the hydrodynamic principle can be applied. The main difference here is that at the static position the contact at the inner bearing occurs at the upper point while the outer journal bearing occurs at the lower point as shown in Fig 1.

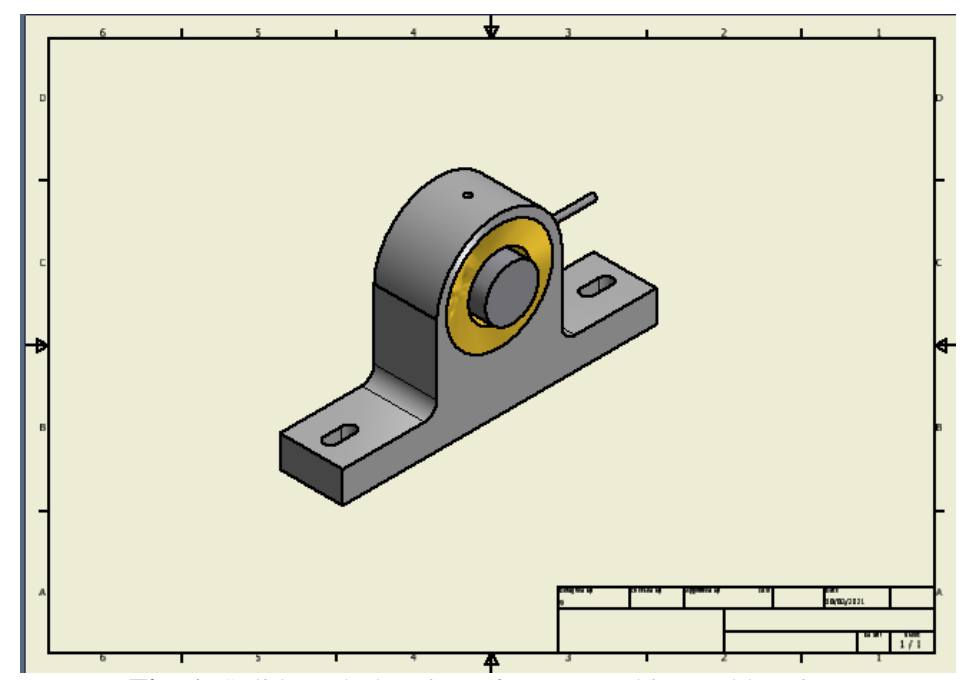

Fig. 1. Solid work drawing of compound journal bearing.

The schematics representation of the compound journal is shown in Fig 2. As it is clear from the figure two pressure profiles are generated due to the hydrodynamic effect for the two film thicknesses of the inner and outer bearing parts. Mathematically the two distinct parts of the compound journal can be treated separately. 


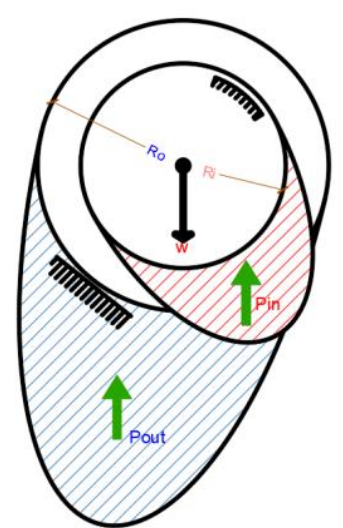

Fig. 2. Pressure profiles of compound journal bearing.

\subsection{Numerical Analysis}

The hydrodynamic Reynolds equation of journal bearing with constant viscosity Newtonian lubricant can be expressed as [1].

$\frac{\partial}{\partial \mathrm{x}}\left(\mathrm{h}^{3} \frac{\partial \mathrm{p}}{\partial \mathrm{x}}\right)+\frac{\partial}{\partial \mathrm{y}}\left(\mathrm{h}^{3} \frac{\partial \mathrm{p}}{\partial \mathrm{y}}\right)=6 \mathrm{U \eta} \frac{\mathrm{dh}}{\mathrm{dx}}$

Where:

$\mathbf{C}, \mathbf{R}, \mathbf{L}$ and $\mathbf{U}$ are bearing radial clearance, radius, axial length and entraining velocity respectively.

$\mathbf{H ,} \mathbf{P}$ and $\eta$ : hydrodynamic film thickness, pressure and dynamic viscosity of the bearing respectively.

Whereas $\mathbf{x}, \mathrm{y}$ are coordinates of hydrodynamic film.

Due to the complexity of the analytical solution of the differential equation for real-sized journals unless in some cases such as for short or very long cases [5]. The main aim of the numerical analysis is to evaluate the main journal parameters such as maximum pressure, load capacity, friction force. In its non-dimensional form, the Reynolds equation is [6]:

$\frac{\partial}{\partial \mathbf{x}^{*}}\left(\mathrm{~h}^{* 3} \frac{\partial \mathbf{p} *}{\partial \mathbf{x}^{*}}\right)+\left(\frac{\mathrm{R}}{\mathrm{L}}\right)^{2} \frac{\partial}{\partial \mathbf{y}^{*}}\left(\mathrm{~h}^{* 3} \frac{\partial \mathbf{p} *}{\partial \mathbf{y}^{*}}\right)=\frac{\partial \mathrm{h}^{*}}{\partial \mathbf{x}^{*}}$

Where;

$\mathrm{h}^{*}=\frac{h}{c} \cdot \mathbf{x}^{*}=\frac{x}{R}, \mathbf{y}^{*}=\frac{y}{L}, \mathrm{p}^{*}=\frac{p c 2}{6 U \eta R}$

The Vogelpohl parameter was invented in the 1930s by Vogelpohl [1] to increase the accuracy of numerical solutions of the Reynolds equation. $\mathrm{Mv}$ is the Vogelpohl parameter, and its definition is as follows: 
$\mathrm{Mv}=\mathrm{p}^{*} \mathrm{~h}^{* 1.5}$

Substituting into eq. 2 one gets

$\frac{\partial^{2} M_{v}}{\partial \chi^{2}}+\left(\frac{R}{L}\right)^{2} \frac{\partial^{2} M_{v}}{\partial \gamma^{2}}=F M_{v}+G$

Where;

$F=\frac{0.75\left[\left(\frac{\partial \lambda}{\partial \chi}\right)^{2}+\left(\frac{R}{L}\right)^{2}\left(\frac{\partial \zeta}{\partial \gamma}\right)^{2}\right]}{\zeta^{2}}$

$G=\frac{\left(\frac{\partial \lambda}{\partial \chi}\right)}{\zeta^{1.5}}$

'Finite difference' approaches are typically used to solve journal and pad bearing difficulties, while 'Finite element' methods have also been used [9]. The finite difference approach works by calculating the difference between function values at two or more consecutive nodes to approximate a differential quantity. By subtracting the formula for $\partial \mathrm{Mv} / \partial \mathrm{x}^{*}$ at the $\mathrm{i}-0.5$ nodal location from the $\mathrm{i}+0.5$ nodal position and dividing by $\delta \mathrm{x}^{*}$, the second differential $22 \mathrm{Mv} / \partial \mathrm{x} * 2$ is found:

$\left(\frac{\partial^{2} \mu_{v}}{\partial \chi^{* 2}}\right)_{i}=\frac{\mu_{v, i+1}+\mu_{v, i-1}-2 \mu_{v, i}}{(\Delta \chi)^{2}}$

Substitute into eq.4 to get.

$\mu_{v, i, j}=\frac{\left(\frac{1}{(\Delta \chi)^{2}}\right)\left(\mu_{v, i+1, j}+\mu_{v, i-1, j}\right)+\left(\frac{R}{L}\right)^{2}\left(\frac{1}{(\Delta \gamma)^{2}}\right)\left(\mu_{v, i+1, j}+\mu_{v, i-1, j}\right)-G_{i, j}}{2 \frac{1}{(\Delta \chi)^{2}}+2 \frac{1}{(\Delta \gamma)^{2}}+F_{i, j}}$

The finite difference approach for solving the Reynolds equation is based on this expression. Its solution yields the necessary Mv nodal values [7].

MATLAB software is introduced to solve eq 10 and evaluating the journal bearing parameters.

\subsection{Bearing Parameters}

The main parameter to be investigated are as follows:

\subsubsection{Pressure Distribution}

The numerical solution of the Reynolds equation can give the pressure distribution at the nodes [8]. The resulting pressure in dimensionless the actual pressure can be found from the following equation.

$\mathrm{p}=\mathrm{p}^{*}(6 \mathrm{U} \eta \mathrm{R}) / \mathrm{C}^{2}$

\subsubsection{Load Capacity}


Integrating the pressure surrounding the bearing yields the total load that the bearing can support. The Half-Sommerfeld condition was utilised in the early literature for load estimates, which meant that the negative pressures in one-half of the bearing were discounted. The load is frequently divided into two parts, one acting parallel to the shaft and bush centres and the other acting perpendicular to the first [12].

$\mathrm{W}=\mathrm{W}^{*}\left(\frac{6 \mathrm{R}^{2} \mathrm{LU} \eta}{c^{2}}\right)$

\subsubsection{Friction Force}

By integrating the shear stress ' $\tau$ ' throughout the bearing area, the friction force may be determined [19]:

$F=\int_{0}^{L} \int_{0}^{2 \pi R} \tau d x d y$

where the shear stress ' $\tau$ ' is given by:

$\tau=\frac{\eta U}{h}+\frac{h}{2} \frac{d p}{d h}$

$\tau=\frac{1}{h *}+3 \mathrm{~h} * \frac{d p *}{d x *}$

and equation (11) can be reduced to:

$\mathrm{F}=\mathrm{F}^{*}\left(\frac{R L \eta U}{c}\right)$

\subsubsection{Coefficient of Friction}

The coefficient of friction of a bearing is calculated once the load and friction forces are Known [16]:

$\mu=\frac{\mathrm{F}}{\mathrm{W}}=\frac{F *\left(\frac{R L \eta U}{c}\right)}{W *\left(\frac{6 R^{2} L U \eta}{c^{2}}\right)}=\left(\frac{C}{6 R}\right)\left(\frac{F *}{W *}\right)$

\subsubsection{Bearing Geometry}

Journal bearings are analysed using the same method as the linear and converging-diverging wedges previously discussed. After defining the film shape, the Reynolds equation is used to calculate the pressure field and load capacity [18].

$\mathrm{h}=\mathrm{c}(1+\varepsilon \cos \theta)$

\section{Experimental Work}




\subsection{Compound Journal Bearing Manufacturing}

The new design of the journal bearing was manufacturing in Middle Technical University (MTU) By using CNC Machen as shown in figure 3. The new design of journal bearing was design to meet the need of the machine with high load capacity, The double journal bearing can be expected to bear more load with less wearing.
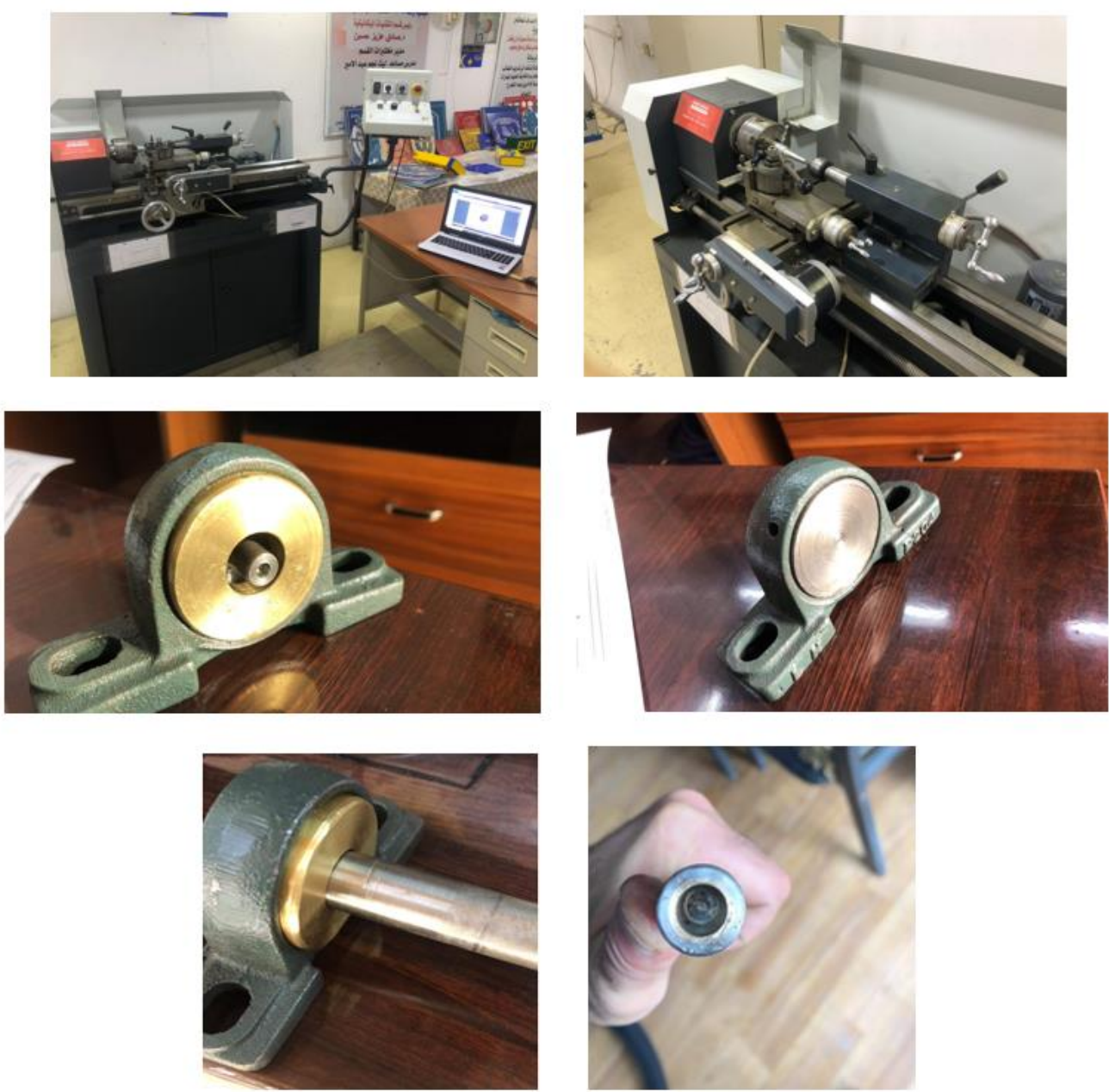

Fig. 3. manufacturing process of new design prototype of compound journal bearing using high accuracy cutting tools machine.

\subsection{Testing Rig}

The testing rig of the double journal bearing is designed to meet the experiments recommend, Fig 4 shown the testing rig. The testing rig has a three-phase motor with a speed reach of 4000 rpm. One side has been fixing with normal journal bearing and the other side compound journal. 
The displacement has been measured with an eddy current sensor with a sensitive reach of 1 micrometre. Fig 5 shows the eddy current sensor. The sensor is attached to amplifier the signal.

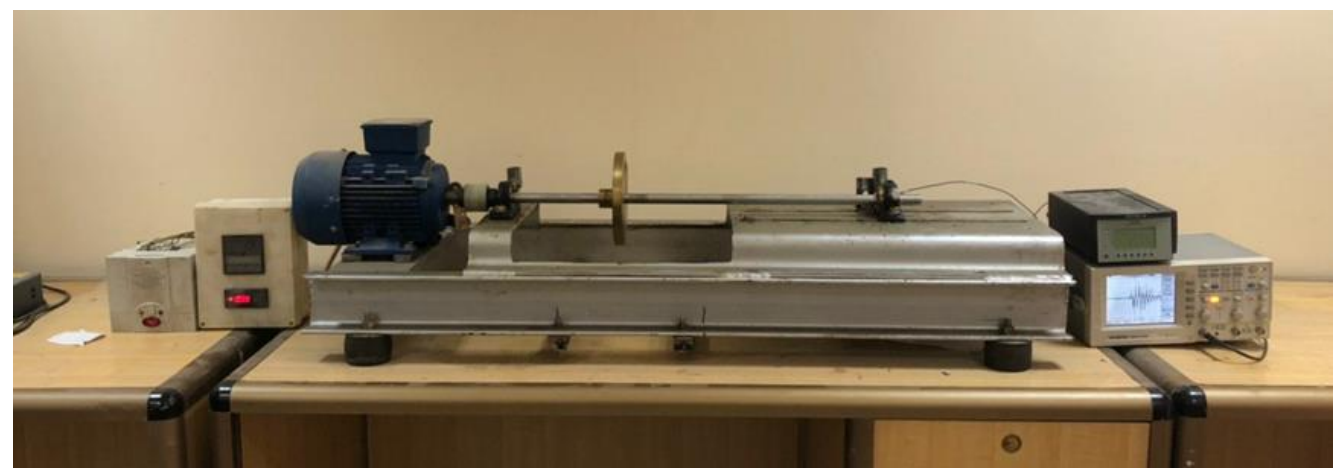

Fig 4. Testing rig.

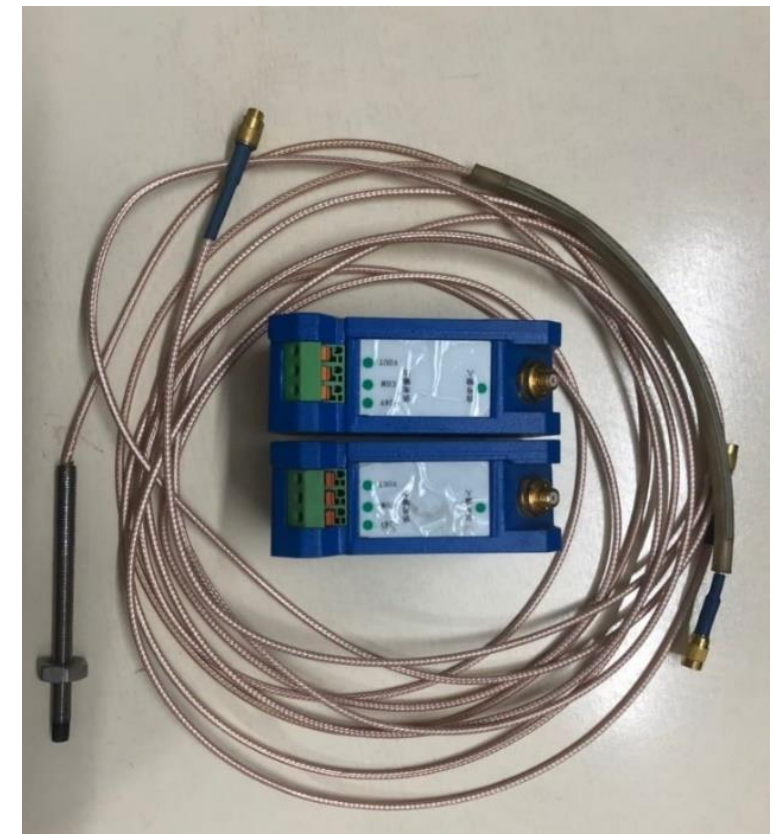

Fig. 5. Eddy's current sensor.

The amplifier is attached to the oscilloscope; as shown in Fig.. 5. The signal of the oscilloscope is transferred to the computer for analysis. The signal is shown in Fig. 6. 


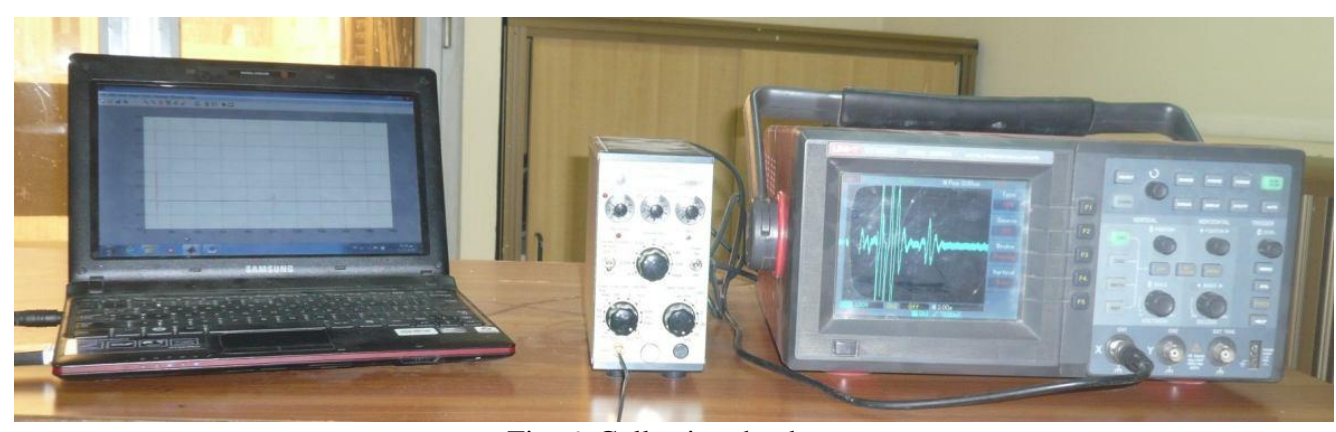

Fig. 6. Collecting the data.

\subsection{Shaft Alignment}

Shaft alignment is required to ensure that the rotational centres of the driver and drive shafts are aligned when the machines are in regular operation. As indicated in Fig 8, misalignment has two components: offset (or parallel) and angular (or face). The distance between the shaft centres of rotation measured in the plane of power transmission from the driving unit to the driven unit is the offset misalignment. The coupling centre is usually where this is measured. The angular misalignment, on the other hand, is the difference in the slope of one shaft (the moveable part) compared to the slope of the other machine's shaft (the stationary part). As already indicated.

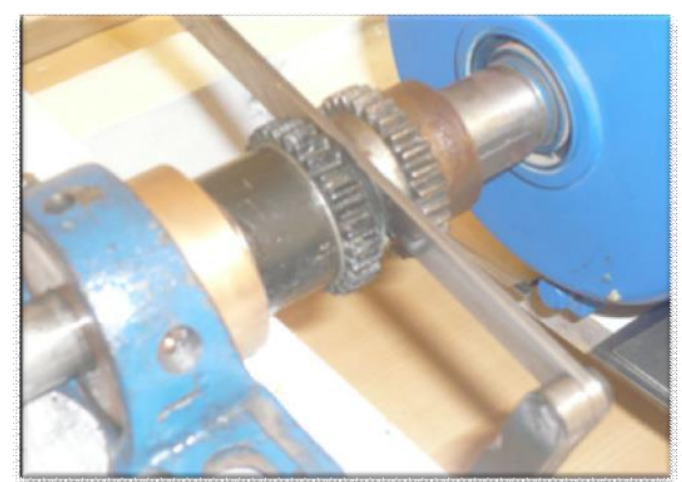

Fig 7. Face measurement by using failure gauge.

\subsection{Experimental Procedures}

The following steps are made for testing and measuring the journal bearing.

1- Selecting the motor speed by adjusting the speed control.

2- Checking the system alignment to avoid any vibration and noise.

3- Reading the output of the proximate sensor probe.

4- Transferring the real-time data 1 to oscilloscope and computer to analysis the signal. and find the associated pressure 
5- Repeating the procedures for many points and different rotating speeds.

\section{Results and Discussion}

To compare the improvement of the new design journal bearing the pressure distribution for the normal and compound bearing are solved and plotted in Figs 8 and 9. These figures show that the pressure profile and the maximum pressure increased to about $50 \%$ for the compound bearing.

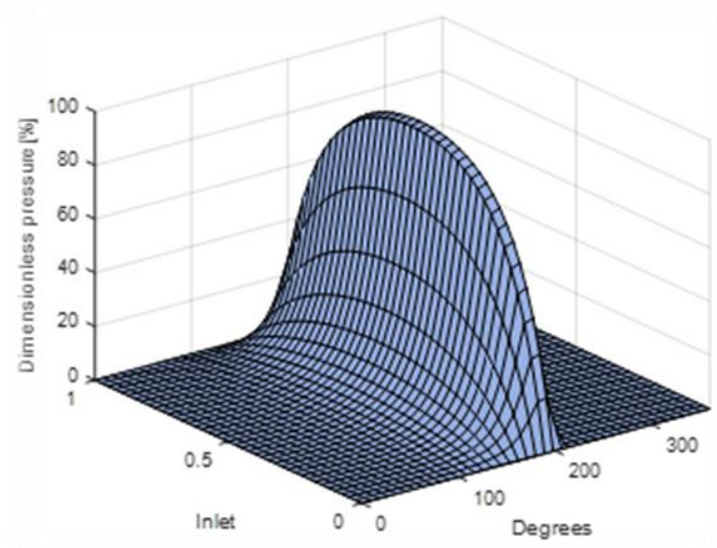

Fig 8. Pressure distribution of normal journal bearing.

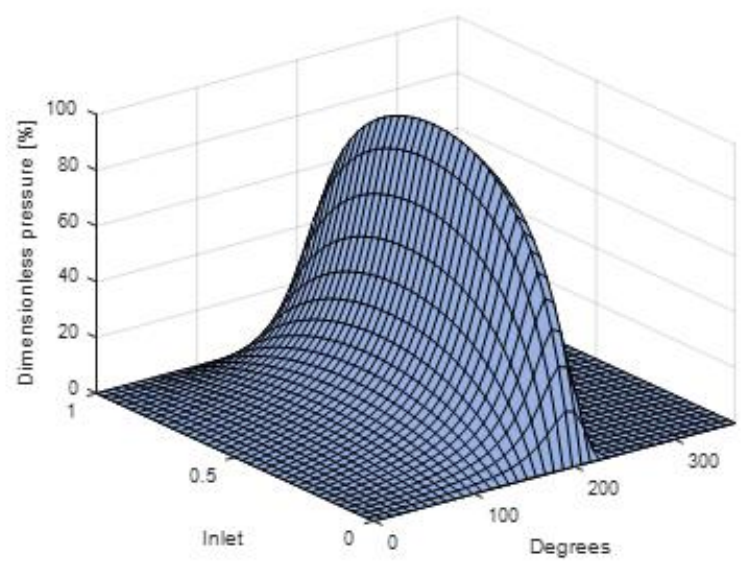

Fig 9. pressure distribution of compound journal bearing. 
The effects of change of eccentricity ratio from 0.5 to 0.9 with $\mathrm{L} / \mathrm{D}=1$ is studied and presented in Table 1. it is clear the load capacity and maximum pressure are increased as the eccentricity ratio increases while both attitude angle and coefficient of friction are decreased.

Table 1. Effect of change of eccentricity ratio of compound bearing.

\begin{tabular}{|c|c|c|c|c|c|}
\hline Case & $\mathrm{e}$ & $\mathrm{W}(\mathrm{kg})$ & $\mathrm{P}(\mathrm{N} / \mathrm{m})$ & $\beta$ & $\mu$ \\
\hline 1 & 0.5 & 0.2962 & 0.33034 & $58.758^{\circ}$ & 43.199 \\
\hline 2 & 0.6 & 0.43644 & 0.52964 & $52.099^{\circ}$ & 40.43 \\
\hline 3 & 0.7 & 0.67912 & 0.92012 & $44.856^{\circ}$ & 27.175 \\
\hline 4 & 0.8 & 1.1894 & 1.8762 & $36.714^{\circ}$ & 11.317 \\
\hline 5 & 0.9 & 2.8044 & 5.4389 & $26.888^{\circ}$ & 5.397 \\
\hline
\end{tabular}

The effect of L/D ratio for the bearing is studied when the ratio is decreased from 1.6 to 0.6 with constant eccentricity ratio $=0.8$ as presented in Table 2 . The outcomes show a proportional relation between this ratio and load capacity, maximum pressure, and attitude angle while the coefficient of friction is inversely proportional.

Table 2. Effect of change of L/D ratio of compound bearing.

\begin{tabular}{|c|c|c|c|c|c|}
\hline Case & L/D & \multicolumn{1}{|l|}{ W(kg) } & P(N/m) & \multicolumn{1}{|c|}{$\beta$} \\
\hline 1 & 1.6 & 1.6078 & 2.2636 & $40.592^{\circ}$ & 7.0293 \\
\hline 2 & 1.4 & 1.4902 & 2.1712 & $39.567^{\circ}$ & 7.4233 \\
\hline 3 & 1.2 & 1.3572 & 2.0529 & $38.145^{\circ}$ & 7.9469 \\
\hline 4 & 1 & 1.1894 & 1.8762 & 36.714 & 11.317 \\
\hline 5 & 0.8 & 0.97757 & 1.6165 & $35.384^{\circ}$ & 23.8 \\
\hline 6 & 0.6 & 0.72555 & 1.265 & $33.664^{\circ}$ & 69.237 \\
\hline
\end{tabular}




\section{Conclusion}

The following conclusions can be drawn from the discussion of the findings:

1- The suggested compound journal bearing increase the pressure profile and the maximum pressure increased about $50 \%$

2- Raising the eccentricity raises the pressure and load capacity while lowering the friction coefficient.

3- Increasing the L/D ratio lowers pressure and increases load capacity while lowering friction coefficient.

4- The performance of the compound bearing can be modified by proper design for eccentricity and $\mathrm{L} / \mathrm{D}$ ratio.

\section{References}

[1] Gwidon W. Stachowiak. Engineering Tribology. United Kingdom. Butterworth-heinemann Vol. 14 pp.26-31,2017.

[2] F.M. Meng . L. Zhang . Y. Liu . Effect of compound dimple on tribological performances of journal bearing. TribolLett Vol 12, pp. 55-61, 2015.

[3] Etsion I, Halperin G,BrizmerV,KligermanY. Experimental investigation of laser surface textured parallel thrust bearings. Vol.9 pp.295-300, 2004

[4] Lu XB, Khonsari MM .An experimental investigation of dimple effect on the stribeck curve of journal bearings. Vol. 71 pp.80-94, 2016

[5]. A. Birket and J.K. Lancaster, Counterface Effects on the Wear of a Composite Dry-Bearing Liner, Proc. JSLE

Int.Tokyo, Japan, Elsevier, pp. 465-470, Vol.157 , 2019

[6] Ling DT, Li ZP, Xiong SW, Grzina D, Cao J, Wang JQ, et al. Surface texturing of drill bits for adhesion reduction and tool life enhancement, Vol. 67, pp.43-51, 2011.

[7] Wng JD, Han ZL, Chen HS, Chen DR. Drag reduction by dimples on surfaces in plane plane contact lubrication, Vol.10, pp.189-194, 2015.

[8] Kngo S, Sharma RK, Pandey RK. Comparative analysis of textured and grooved hydrodynamic journal bearing. Proc IMechE, Vol. 64, pp.547-552, 2019.

[9] Shen C, Khonsai MM. Effect of dimple's internal structure on hydrodynamic lubrication, Vol.67, pp.104-110, 2014.

[10] Arghir M, Rocou N, Helene M, Frene J. Theoretical analysis of the incom- pressible laminar flow in a macro-roughness cell, Vol.10, pp.241-251, 2013.

[11] Sahin F, Glavatskih SB, Almqvist T, Larsson R. Two-dimensional CFD-analysis of micropatterned surfaces in hydrodynamic lubrication.

[12] ANSYS Help manual, release 14.5, Inc., Pittsburgh, Pennsylvania of USA; 2012.

[13] Cupillrd S, Glavatskih S, Cervantes MJ. Computational fluid dynamics analysis of a journal bearing with surface texturing. Proc Inst IMechE J.

[14] Go GY, Yin ZW, Jiang D, Zhang XL. Numerical analysis of plain journal bearing under hydrodynamic lubrication by water.

[15] Baun D, Greiner C, Schneider J, Gumbsch P. Efficiency of laser surface texturing in the reduction of friction under mixed lubrication.

[16] Etion I, Halperin G, Brizmer V, Kligerman Y. Experimental investigation of laser surface textured parallel thrust bearings. 
[17] Lu XB, Khnsari MM. An experimental investigation of dimple effect on the stribeck curve of journal bearings.

[18] Meg FM, Yang T. Preliminary study on mechanism of cavitation in lubricant of textured sliding bearing. Proc Inst Mech Eng J: J Eng Tribol 2013;227 (7):695-708.

[19] K. G. Binu, B. S. Shenoy, D. S. Rao, and R. Pai, A Variable Viscosity Approach for the Evaluation of Load Carrying Capacity of Oil Lubricated Journal Bearing with TiO2 Nanoparticles as Lubricant Additives, Procedia Mater. Sci., vol. 6, no. Icmpc, pp. 1051-1067, 2014. 